\title{
Fano-like Anti-resonances in Nanomechanical and Optomechanical Systems
}

\author{
D.A. Rodrigues \\ School of Physics and Astronomy, University of Nottingham, Nottingham NGr 2RD, U.K.
}

\begin{abstract}
We study a resonator coupled to a generic detector and calculate the noise spectra of the two sub-systems. We describe the coupled system by a closed, linear, set of Langevin equations and derive a general form for the finite frequency noise of both the resonator and the detector. The resonator spectrum is the well-known thermal form with an effective damping, frequency shift and diffusion term. In contrast, the detector noise shows a rather striking Fano-like resonance, i.e. there is a resonance at the renormalized frequency, and an anti-resonance at the bare resonator frequency. As examples of this effect, we calculate the spectrum of a normal state single electron transistor coupled capacitively to a resonator and of a cavity coupled parametrically to a resonator.
\end{abstract}

PACS numbers: $85.85 .+\mathrm{j}, 85.35 . \mathrm{Gv}, 42.79 . \mathrm{Gn}$

When a mechanical resonator is coupled to a detector, even weakly, the detector can have a significant effect on the dynamics of the resonator, and it is this "backaction" that ultimately enforces the standard quantum limit of measurement [1]. For weak enough coupling, the detector acts like an additional thermal bath, providing an effective frequency shift, damping and temperature. This effective temperature can be lower than that of the resonator's environment, so a detector, such as an optical/microwave cavity or a mesoscopic conductor, can cool the resonator [2, 3], potentially to its ground state (4] and refs. therein). Linear response theory gives a general way of calculating the noise spectrum of a resonator coupled to a detector, which is found to be very close to a thermal spectrum [5]. A relevant question is if a thermal model is enough to fully capture the dynamics of the system, or if there are any effects beyond a purely thermal back action. In particular, when calculating the spectrum of the detector, can we still treat the back action as purely thermal?

In this Letter, we consider a detector linearly coupled to a resonator, and calculate the noise spectrum of both. As expected, the resonator spectrum is essentially thermal, with the frequency, damping and temperature modified by the back-action. We might therefore expect that the detector spectrum is close to the spectrum of a backaction-free detector coupled to a resonator with this modified thermal bath. However, we find that this is not the case, and show that the noise in the detector instead has a rather striking feature akin to a Fano resonance [6], i.e. a resonance at the renormalized resonator frequency plus an anti-resonance at the original frequency. Fano resonances arise from the interference between coherent and incoherent paths in mesoscopic conductors [7], but resonance/anti-resonance pairs are a general interference phenomenon, occurring in systems from LC circuits to electromagnetically induced transparency (EIT) [8, 9].

We first outline our general formalism before deriving an expression for the spectrum of a generic detector coupled to a resonator. Finally, we illustrate the analysis with two examples: a single electron transistor coupled capacitively to a resonator, and a cavity coupled parametrically to a resonator.

We assume that system (resonator plus detector) can be described near a stable steady state by a linear set of Langevin equations,

$$
\dot{\vec{\varrho}}=-\mathbf{A} \vec{\varrho}+\vec{\xi}
$$

where the vector $\vec{\varrho}=\left(x, v, \sigma_{1}, \sigma_{2} \ldots\right)$ includes the resonator position $x$ and velocity $v$, and the degrees of freedom of the detector $\sigma_{i}$. The term $\vec{\varrho}$ can refer to either a set of classical dynamical variables, or a set of Heisenberg operators for the system. The matrix $\mathbf{A}$ describes the evolution of the means of the variables, and $\vec{\xi}$ describes fluctuations about these means. If the equations of motion are non-linear, we can linearize them to obtain the desired form, as long as the fluctuations of the variables about their steady state values are small [11].

The noise in the system can be calculated from Eq. (11) directly, but by considering the sub-systems separately and assuming a specific form for the coupling we find that we can write down a more illuminating general form for both resonator and detector noise. The resonator (either mechanical or a superconducting stripline or coplanar waveguide [3] ) is assumed to be a single-mode harmonic oscillator, whose motion depends on a linear sum of the detector variables, which we denote $\sigma_{n}$, with overall strength $x_{s}$. The resonator variables then obey the equations of motion $\dot{x}=v$ and,

$$
\dot{v}=-\omega_{0}^{2} x-\gamma_{e} v+\omega_{0}^{2} x_{s} \sigma_{n}+\xi_{e},
$$

with $\omega_{0}$ the resonator frequency and $\gamma_{e}, \xi_{e}$ the damping and fluctuations it feels due to its thermal bath.

The detector is described by a set of linear equations for its degrees of freedom, $\sigma_{i}$,

$$
\dot{\vec{\sigma}}=-\mathbf{B} \vec{\sigma}+\vec{\kappa} x+\vec{\xi}_{\sigma} .
$$

Matrix $\mathbf{B}$ gives the $x$-independent evolution of the detector variable means. The rate of change of each variable $\sigma_{i}$ depends on resonator position $x$ with strength $\kappa_{i}$. 
The vector $\vec{\xi}_{\sigma}$ in Eq. (3) describes the fluctuations of $\vec{\sigma}$, which may simply be thermal but can also arise from other stochastic processes (such as incoherent tunneling [10]). In the Markovian approximation, the $\xi$ terms acting on both the resonator and the detector will be $\delta$ correlated, $\left\langle\xi_{i}\right\rangle=0,\left\langle\xi_{i}(t) \xi_{j}\left(t^{\prime}\right)\right\rangle=G_{i j} \delta\left(t-t^{\prime}\right)$. The correlators are related to the variances by [11],

$$
\mathbf{G}=\left(\mathbf{A} \chi+\chi \mathbf{A}^{\mathbf{T}}\right),
$$

with $\chi$ the matrix of steady state variances of the coupled system $\chi_{i j}=\left\langle\varrho_{i} \varrho_{j}\right\rangle-\left\langle\varrho_{i}\right\rangle\left\langle\varrho_{j}\right\rangle$. In a quantum system, the commutation relations can mean that $\chi_{i j} \neq \chi_{j i}$.

With our formalism set up, we solve the equations of motion. Fourier transforming Eq. (3) and solving for $\sigma_{n}$,

$$
\sigma_{n}(\omega)=\vec{n}^{T}(\mathbf{B}+i \omega)^{-1} \vec{\xi}_{\sigma}(\omega)+x(\omega) \vec{n}^{T}(\mathbf{B}+i \omega)^{-1} \vec{\kappa},
$$

where $\vec{n}^{T}$ is the row vector defined by $\vec{n}^{T} \vec{\sigma}=\sigma_{n}$. We substitute this into the Fourier transform of Eq. (2) to get $x(\omega)$. We can then obtain the resonator spectrum, using Eq. (4), the Hermitian nature of the fluctuations, and the fact that noise operators $\delta$-correlated in time will also be $\delta$-correlated in frequency $\left\langle\xi(\omega) \xi\left(\omega^{\prime}\right)\right\rangle \propto \delta\left(\omega+\omega^{\prime}\right)$. Neglecting resonator bath fluctuations $\xi_{e}$,

$$
S_{x x}(\omega)=\frac{S_{v v}(\omega)}{\omega^{2}}=\frac{\omega_{0}^{4} x_{s}^{2} S_{\sigma_{n} \sigma_{n}}^{\prime}(\omega)}{\left(\omega_{r}(\omega)^{2}-\omega^{2}\right)^{2}+\omega^{2} \gamma_{T}(\omega)^{2}} .
$$

This is written in the form of a resonator coupled to a heat bath, defining the renormalized frequency $\omega_{r}^{2}(\omega)=$ $\omega_{0}^{2}+\delta \omega_{0}^{2}(\omega)$ and total damping $\gamma_{T}(\omega)=\gamma_{e}+\gamma_{\sigma}(\omega)$. Noting that the frequency-dependent effective back-action damping $\gamma_{\sigma}(\omega)$ and frequency shift $\delta \omega_{0}^{2}(\omega)$ terms are real, these expressions are given by [12],

$$
\delta \omega_{0}^{2}(\omega)+i \omega \gamma_{\sigma}(\omega)=-\omega_{0}^{2} x_{s} \vec{n}^{T}(\mathbf{B}+i \omega)^{-1} \vec{\kappa} .
$$

$S_{\sigma_{n} \sigma_{n}}^{\prime}(\omega)$ is closely related to $S_{\sigma_{n} \sigma_{n}}^{0}(\omega)$, the zero-coupling spectrum of $\sigma_{n}$, and is given by,

$$
\mathbf{S}_{\sigma \sigma}^{\prime}(\omega)=(\mathbf{B}+i \omega)^{-1} \mathbf{G}_{\sigma \sigma}\left(\mathbf{B}^{\mathbf{T}}-i \omega\right)^{-1}
$$

where $\mathbf{G}_{\sigma \sigma}$ refers to the subset of $\mathbf{G}$ that describes the detector variables. The resonator can modify $\mathbf{G}_{\sigma \sigma}$ and linearization can renormalize $\mathbf{B}$, but for weak enough coupling both these effects will be small. If we can approximate these terms by their uncoupled values, then we have $S_{\sigma_{n} \sigma_{n}}^{\prime}(\omega) \approx S_{\sigma_{n} \sigma_{n}}^{0}(\omega)$, and the noise on the resonator reduces to the standard linear response expression. Equation (6) describes a thermal-like spectrum, where weak coupling implies a narrow resonance, so we can approximate the $\omega$-dependent terms by their values at $\omega_{r} \approx \omega_{0}$, and define a diffusion term $D_{\sigma}=\omega_{0}^{4} x_{s}^{2} S_{\sigma_{n} \sigma_{n}}^{\prime}\left(\omega_{0}\right)$.

The effect of the detector back-action on the resonator spectrum is essentially just a modification of the thermal parameters. We might therefore expect the detector spectrum to be basically that of a back-action-free detector coupled to a resonator with modified thermal parameters, $\omega_{r}, \gamma_{T}, D_{\sigma}$. However, we find that that this is not the case, and that the detector noise can be strongly modified from this naive picture,

$$
S_{\sigma_{n} \sigma_{n}}(\omega)=\frac{\left(\omega_{0}^{2}-\omega^{2}\right)^{2}+\omega^{2} \gamma_{e}^{2}}{\left(\omega_{r}(\omega)^{2}-\omega^{2}\right)^{2}+\omega^{2} \gamma_{T}(\omega)^{2}} S_{\sigma_{n} \sigma_{n}}^{\prime}(\omega) .
$$

The spectrum of the detector noise has a Fano-like resonance [6, 13], i.e. there is a resonance at the renormalised frequency $\omega= \pm \omega_{r}(\omega)$ and an anti-resonance at the unrenormalised frequency $\omega= \pm \omega_{0}$. Somewhat surprisingly, we find that if $\gamma_{e}=0$ the noise at the resonator frequency is exactly zero, i.e. $S_{\sigma_{n} \sigma_{n}}\left( \pm \omega_{0}\right)=0$, independent of the parameters of the detector. Just as the spectrum of the resonator near $\omega_{0}$ is captured by three parameters, $\omega_{r}, \gamma_{T}, D_{\sigma}$, the spectrum of $\sigma_{n}$ only requires the additional two parameters $\omega_{0}, \gamma_{e}$.

The anti-resonance in the detector noise can be understood in a simple, intuitive way [8]. The detector noise at frequency $\omega$ depends on how sensitive the detector is to an external oscillating force at that frequency. For our coupled system, a perturbation on the detector at frequency $\omega$ will also cause the resonator to respond at $\omega$. The detector then feels the perturbation in two ways: the original force, and a corresponding force from the resonator. The resonator force is exactly out of phase with the external perturbation and so acts to cancel it. When $\gamma_{e}=0$, the forces exactly cancel and the noise goes to zero at $\omega=\omega_{0}$. Thus the anti-resonance can be understood as classical interference, analogous to quantum interference in mesoscopic conductors [7] or EIT 8].

We also see why the sub-systems have such a different effect on each other. The detector relaxes quickly (compared to the resonator damping), so has a short "memory" and acts like a Markovian thermal bath for the resonator. In contrast, weak damping $\gamma_{e}$ means the resonator has a long memory, so the detector sees a highly non-Markovian bath and has a non-thermal spectrum.

Although no approximations have been made in deriving Eqs. (6) and (9) from Eq. (11), $\gamma_{\sigma}, \delta \omega_{0}^{2}$ and $S_{\sigma_{n} \sigma_{n}}^{\prime}$ are all $\omega$-dependent, so generally Eqs. (6) and (9) will not look like thermal and Fano spectra. These emerge only when the coupling between the resonator and detector is weak enough that the resonance is narrow on scale of the change in these parameters, i.e. $\gamma_{T}\left(\omega_{0}\right)$ must be much smaller than the dissipative terms on the detector.

We now include thermal fluctuations from the resonator's environment. Assuming that the resonator's bath is unaffected by the detector, Eq. (4) shows that the bath flucuations $\xi_{e}$ obey the standard expressions, $\left\langle\xi_{e}\right\rangle=0,\left\langle\xi_{e}(t) \xi_{e}\left(t^{\prime}\right)\right\rangle=\delta\left(t-t^{\prime}\right) D_{e}$, where $D_{e}$ is the diffusion. We also require the correlations between the fluctuation terms on the resonator $\xi_{e}$ and on the detector $\xi_{i}$. If these terms vanish $\left\langle\xi_{e}(t) \xi_{i}\left(t^{\prime}\right)\right\rangle=0$, (typically the 
case [14]), the resonator is described by Eq. (6) with a total diffusion $D_{T}=D_{\sigma}+D_{e}$. The detector spectrum is,

$$
\begin{aligned}
S_{\sigma_{n} \sigma_{n}}(\omega)= & \frac{\left(\omega_{0}^{2}-\omega^{2}\right)^{2}+\omega^{2} \gamma_{e}^{2}}{\left(\omega_{r}(\omega)^{2}-\omega^{2}\right)^{2}+\omega^{2} \gamma_{T}(\omega)^{2}} S_{\sigma_{n} \sigma_{n}}^{\prime}(\omega) \\
& +\frac{\delta \omega_{0}^{2}(\omega)^{2}+\omega^{2} \gamma_{\sigma}(\omega)^{2}}{\left(\omega_{r}(\omega)^{2}-\omega^{2}\right)^{2}+\omega^{2} \gamma_{T}(\omega)^{2}} \frac{D_{e}}{\omega_{0}^{4} x_{s}^{2}} .
\end{aligned}
$$

The detector noise at $\omega_{0}$ is suppressed below the uncoupled value by the factor $R=S_{\sigma_{n} \sigma_{n}}\left(\omega_{0}\right) / S_{\sigma_{n} \sigma_{n}}^{0}\left(\omega_{0}\right) \sim$ $\left(\gamma_{e}^{2}+\gamma_{\sigma}^{2} \frac{D_{e}}{D_{\sigma}}\right) / \gamma_{T}^{2}$. Thus $\gamma_{e}^{2} / \gamma_{\sigma}^{2} \ll 1$ is a neccessary condition for $R \ll 1$. In terms of the effective thermal occupation numbers, when the back action is strong enough that $\frac{n_{\sigma}}{n_{e}} \gg \frac{\gamma_{\sigma}}{\gamma_{e}} \gg 1$, Eq. (10) reduces to Eq. (9) and $R \approx$ $\gamma_{e}^{2} / \gamma_{\sigma}^{2} \ll 1$. In the opposite regime $n_{\sigma} \lesssim n_{e}, \gamma_{e} \ll \gamma_{\sigma}$, the suppression of the noise is given by $R \approx \frac{\gamma_{e}\left(2 n_{e}+1\right)}{\gamma_{\sigma}\left(2 n_{\sigma}+1\right)}$. So, the anti-resonance is only present if the back action dominates, i.e. $n_{\sigma} \gg n_{e} \gamma_{\sigma} / \gamma_{e}$, or the parameters allow ground state cooling. Thus, the effect will be hard to observe when the the resonator is cooled from a very high ambient temperature, as is often the case with optical frequency cavities. Fig. 1(a) inset (iii) shows the effect of including the environment using parameters from Ref. [2], (where $\gamma_{e} \approx \gamma_{\sigma}, T_{\sigma} \approx 100 \mathrm{mK}$ and $T_{e} \lesssim 600 \mathrm{mK}$ ).

We now illustrate this formalism by two well-known systems, a resonator coupled to a normal-state SET, and to an optical or microwave cavity. In the SET case 15, 17], the resonator position couples to a single operator describing the charge on the island, $\sigma=|1\rangle\langle 1|$, where $|1\rangle$ is the state with one extra charge on the island. If the junction resistances are equal, we have a single, closed, linear equation of motion,

$$
\dot{\sigma}=\Gamma_{R}-\left(\Gamma_{L}+\Gamma_{R}\right) \sigma+\kappa x+\xi_{\sigma},
$$

where $\Gamma_{L}$ and $\Gamma_{R}$ are the tunnel rates across the left and right junctions, respectively, which can be varied by altering the gate voltage. To get the equations of motion in the form of Eq. (1), we shift to variables describing deviations from the steady state, $\sigma^{\prime}=\sigma-\langle\sigma\rangle$, and $x^{\prime}=x-\langle x\rangle$. The evolution matrix $\mathbf{B}=\Gamma_{L}+\Gamma_{R}=\Gamma_{T}$ and the coupling and fluctuation vectors $\vec{\kappa}=\kappa, \vec{\xi}_{\sigma}=\xi_{\sigma}$ are simply scalars. Equation (7) then gives a simple expression for the damping and frequency shift, $\delta \omega_{0}^{2}(\omega)+i \omega \gamma_{\sigma}(\omega)=$ $-\omega_{0}^{2} x_{s} \kappa\left(\Gamma_{T}-i \omega\right) /\left(\Gamma_{T}^{2}+\omega^{2}\right)$ and we use Eq. (44), or the evolution of the first and second moments [10], to obtain,

$$
G_{\sigma \sigma}=\Gamma_{R}(1-2\langle\sigma\rangle)+\Gamma_{T}\langle\sigma\rangle+\kappa(\langle x\rangle-2\langle x \sigma\rangle) .
$$

This leads to the position and charge noise spectra, plotted in Fig. 1(a). The spectra will be thermal and Fanolike as long as weak coupling holds $\Gamma_{L, R} \gg \gamma_{T}\left(\omega_{0}\right)$.

The SET-resonator equations are closed and linear to start with [15, 17]. In contrast, the equations of motion for a cavity coupled to a resonator [16] are non-linear. We can still apply our formalism if we linearize these
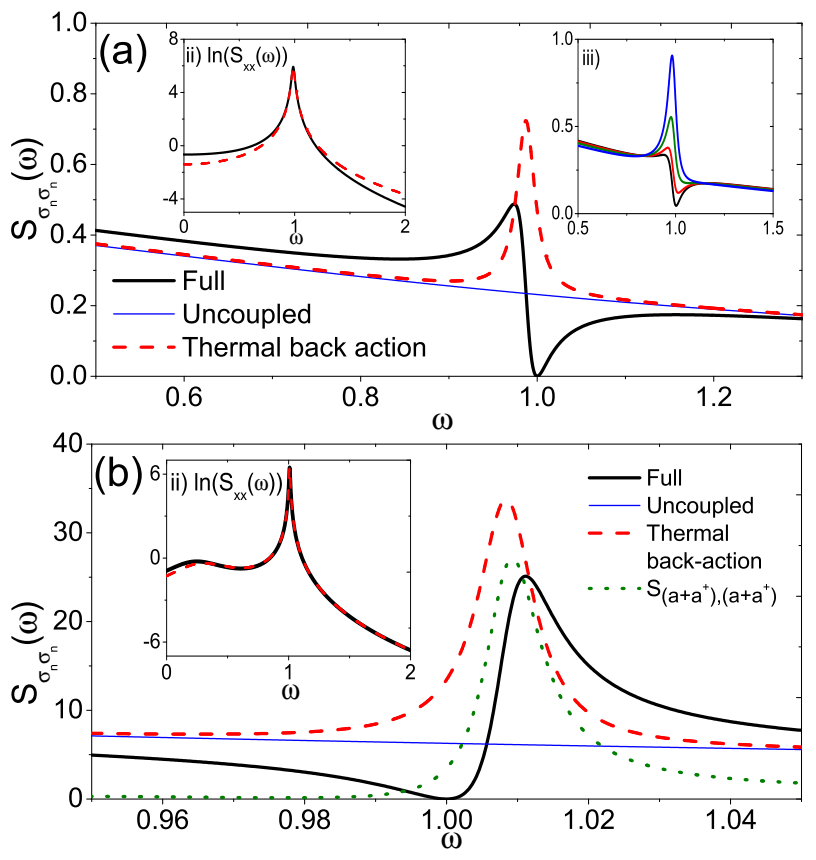

FIG. 1: (color online). Fano-like anti-resonances in the spectra of an $\operatorname{SET}(\mathrm{a})$ and a cavity(b) coupled to a resonator. Shown is detector noise in the fully coupled system (solid line), when the coupling is zero (thin line) and when the back action is assumed to be purely thermal (dashed line). The thermal model fails to capture the anti-resonance, although it matches the resonator noise very well (insets $i$, logarithmic scale). The SET plot also shows the effect of environmental diffusion and damping for experimentally relevant values (iii) [2]. The noise of a general quadrature $a+a^{\dagger}$ (rather than $\left.\sigma_{n}=a^{\prime}\left\langle a^{\dagger}\right\rangle+a^{\prime \dagger}\langle a\rangle\right)$ shows a signature of the antiresonance in its asymmetric peak (dotted). SET parameters: $\Gamma_{L}=0.6, \Gamma_{R}=0.4, \omega_{0}=1, \kappa=0.05$ and $x_{s}=1$; for the cavity: $\Delta=0.3, \gamma_{a}=0.5, g=0.05, \omega_{0}=1, N_{a}=0$ and $\alpha=1$; for both main plots $\gamma_{e}$ and $D_{e}=0$ and for inset (iii) (a), $\gamma_{e} / \gamma_{\sigma}=1$ and $D_{e} / D_{\sigma}=6,3,1$ and 0 from top to bottom [2].

equations about their steady state values $\langle x\rangle,\langle a\rangle$. With shifted variables $x^{\prime}=x-\langle x\rangle, a^{\prime}=a-\langle a\rangle$ and cavity detuning and damping, $\Delta, \gamma_{a}$,

$$
\begin{aligned}
& \dot{v}^{\prime}=-\omega_{0}^{2} x^{\prime}-\gamma_{e} v^{\prime}-2 g\left(\left\langle a^{\dagger}\right\rangle a^{\prime}+a^{\prime \dagger}\langle a\rangle\right)+\xi_{e} \\
& \dot{a}^{\prime}=i(\Delta+g\langle x\rangle) a^{\prime}-\frac{\gamma_{a}}{2} a^{\prime}+i g x^{\prime}\langle a\rangle+\xi_{a} .
\end{aligned}
$$

is obtained, plus the complex conjugate equation for the cavity field $a^{\prime \dagger}$.

The steady state values simply act as parameters, so Eq. (13) has the same form as Eq. (21). The variable $\sigma_{n}=\left\langle a^{\dagger}\right\rangle a^{\prime}+\langle a\rangle a^{\prime \dagger}$ is a particular quadrature of the field (determined by the driving laser amplitude $\alpha$ ), which affects the resonator with a strength determined by $x_{s}=2 g / \omega_{0}^{2}$. We can also rewrite Eq. (14) and its complex conjugate in the form of Eq. (3) for the cavity variables $\vec{\sigma}=\left(\begin{array}{c}a^{\prime} \\ a^{\prime \dagger}\end{array}\right) . \quad \mathbf{B}=\left(\begin{array}{cc}B^{-} & 0 \\ 0 & B^{+}\end{array}\right)$determines their mean evolution, where $B^{ \pm}=\frac{\gamma_{a}}{2} \pm i(\Delta+g\langle x\rangle)$, and 
$\vec{\kappa}=i g\left(\begin{array}{c}\langle a\rangle \\ -\left\langle a^{\dagger}\right\rangle\end{array}\right)$, with $\vec{n}^{T}=\left(\left\langle a^{\dagger}\right\rangle,\langle a\rangle\right)$. Cavity fluctuations are described by the standard terms, $G_{a^{\dagger} a}=\gamma_{a} N_{a}$, $G_{a a^{\dagger}}=\gamma_{a}\left(N_{a}+1\right)$ where $N_{a}$ gives the cavity temperature. We insert $\mathbf{B}, \mathbf{G}_{\sigma \sigma}, \vec{n}^{T}$ and $\vec{\kappa}$ into Eq. (10) to obtain the spectra. Weak-coupling holds when $\gamma_{a} \gg \gamma_{T}\left(\omega_{0}\right)$ : the cavity is damped much more rapidly than the resonator.

Figure 1 shows the spectra of $x$ and $\sigma_{n}$ for the SET resonator and the cavity resonator. As a comparison, we also plot the spectra of a back-action free detector measuring a thermal resonator with modified parameters [15]. To calculate this we eliminate the back-action term $\omega_{0}^{2} x_{s} \sigma_{n}$ in Eq. (2), calculate the spectra, and then replace the thermal parameters with the values they would have due to back-action (we set $\omega_{0}=\omega_{r}, \gamma_{e}=\gamma_{T}, D_{e}=D_{T}$ ). The resonator spectrum is very well matched, deviation only occurring far from resonance where the noise is essentially zero. In contrast, the thermal backaction model completely fails to capture the anti-resonance in the device spectrum. A similar antiresonance will occur for any such "detector" weakly coupled to a resonator, if $\gamma_{e}$ and $D_{e}$ are low enough.

The frequency spectrum of the detector is a major way of obtaining information about the dynamics of the resonator. Although the antiresonance occurs in one particular detector variable $\sigma_{n}$ (which might not be the most experimentally relevant), it should be possible to see signatures in other observables, e.g. a dip in SET current (not charge) noise [15, 18].

Input-output theory [1] $b_{\text {out }}=b_{\text {in }}+\sqrt{\gamma_{a}} a^{\prime} / 2$ shows the spectral properties of the cavity are directly transferred to the output field, and hence detectable via a homodyne measurement of a field quadrature. If $R \gtrsim 1$, the output spectrum is well approximated by the resonator spectrum multiplied by a constant [16]. If the back-action noise is not negligible, it will be modified to a Fano-like form. The spectrum will only take the exact form Eq. (10) when the quadrature has the same phase as $\sigma_{n}$, but the spectra of other quadratures will show signatures of the Fano resonance, e.g. an asymmetric peak (Fig. 1).

A detector spectrum showing a Fano-lineshape can reveal more about the dynamics of the resonator; the bare as well as renormalized frequencies and both the total and environmental damping. Fano resonances could lead to more sensitive measurements and applications such as transmission-line switches and analogues of EIT [19].

In conclusion, we studied the spectra of a detector and resonator coupled in a particular (but quite general) way. The detector variables depend linearly on $x$, and the back action force is proportional to a particular detector variable $\sigma_{n}$. If the system can be described by linear Langevin equations, the spectra of the resonator and the detector variable $\sigma_{n}$ can easily be derived. In the weak-coupling regime where the dissipation of the detector is much larger than the total resonator damp- ing (e.g. $\gamma_{a} \gg \gamma_{T}$ ), these spectra reduce to a generic form. The resonator spectrum is approximately thermal, but the spectrum of the detector variable coupled to the resonator has a resonance at the renormalized frequency and an anti-resonance at the bare resonator frequency. We calculated the spectra of a resonator coupled capacitively to a normal state SET, and parametrically to a resonator. The detector spectra differ significantly from those derived from a purely thermal back-action model.

We acknowledge helpful discussions with Andrew Armour, Steve Bennet, Miles Blencowe, Aashish Clerk and Alex Rimberg, and funding by EPSRC grant $\mathrm{EP} / \mathrm{D} 066417 / 1$.

[1] C. M. Caves, Phys. Rev. Lett. 45, 75 (1980).

[2] A. Naik et. al., Nature 443, 193(2006); O. Astafiev et. al., Nature 449588 (2007).

[3] S. Gigan et. al. Nature 444, 67 (2006); O. Arcizet et. al. Nature 444, 71 (2006); D. Kleckner and D. Bouwmeester, Nature 444, 75 (2006); C. A. Regal, J. D. Teufel and K. W. Lehnert, Nature Physics 4, 555 (2008); A. Schliesser et. al, Nature Physics 4, 415 (2008).

[4] F. Marquardt, A. A. Clerk, S. M. Girvin, arXiv:0803.1164

[5] A. A. Clerk, Phys. Rev. B 70, 245306 (2004).

[6] U. Fano, Phys. Rev. 124, 1866 (1961).

[7] A. A. Clerk, X. Waintal and P. W. Brouwer, Phys. Rev. Lett. 86, 4636 (2001).

[8] C. L. Garrido Alzar, M. A. G. Martinez and P. Nussenzveig, Am. J. Phys 70, 41 (2002).

[9] M. Fleischauer, A. Imamoglu and J. P. Marangos, Rev. Mod. Phys. 77, 633 (2005).

[10] D. A. Rodrigues and G. J. Milburn, Phys. Rev. B, 78 104302 (2008).

[11] D. F. Walls and G. J. Milburn 1994 Quantum Optics (Berlin: Springer-Verlag).

[12] If the master equations of the sub-systems are simply coupled by a Hamiltonian term, Eq. (7) equals the antisymmetrized noise, but is also valid if the dissipative terms are modified (e.g. $x$-dependent tunnel rates).

[13] Eq. (9) has resonances at $\pm \omega_{r}$, not only $\omega_{r}$ [6], as we have not made the RWA, valid for a slowly relaxing detector.

[14] Often holds, e.g. master equations coupled by a Hamiltonian term or $x$-dependent tunneling.

[15] A. D. Armour, M. P. Blencowe and Y. Zhang, Phys. Rev. B, 69125313 (2004); A. D. Armour, Phys. Rev. B, 70 165315 (2004); D. A. Rodrigues and A. D. Armour, New. J. Phys. 7, 251 (2005).

[16] F. Marquardt et. al., Phys. Rev. Lett. 99, 093902 (2007); I. Wilson-Rae, et. al., Phys. Rev. Lett. 99, 093901 (2007); A. Dantan, et. al., Phys. Rev. A 77, 011804(R) (2008); M. Paternostro, et. al., New J. Phys. 8, 107 (2006).

[17] A. A. Clerk and S. D. Bennett, New J. Phys. 7, 238 (2005).

[18] V. Koerting et. al. arXiv:0810.5718.

[19] V. Vyurkov, L. Gorelik, and A. Orlikovsky, arXiv:0806.4339; L. Zhou et. al., Phys. Rev. Lett. 101, 100501 (2008); X-Z. Yuan et. al. New J. Phys. 10, 095016 (2008). 